TABLE I

\begin{tabular}{lcc}
\multicolumn{3}{c}{ Blood Transfusion In CaEsarian SeCtion Patients } \\
\hline & Number & Per cent \\
\hline Total caesarian sections & 430 & 100 \\
Preoperative transfusions (> 12 hr preoperatively) & & \\
- elective sections - anaemia & 2 & 0.5 \\
- placenta praevia & 2 & 0.5 \\
Perioperative transfusions & 3 & \\
- bleeding preoperatively - abruptio & 3 & 0.7 \\
- bleeding preoperatively - praevia & 3 & 0.7 \\
- no bleeding preoperatively - uterine tears & & \\
Postoperative transfusions (>48 hrs postpartum) & 3 & 0.7 \\
- anaemia (Hb 8-9 gm\%) & 1 & 0.2 \\
- delayed postpartum haemorrhage & 16 & 3.7 \\
Total patients transfused &
\end{tabular}

( 0.5 per cent of all sections). These patients received crystalloid in the operating room and crystalloid plus packed cells in the recovery room. Before any blood was transfused, one patient had a blood pressure of 120 systolic and a heart rate of $100 / \mathrm{min}$.; the other had a blood pressure of 100 systolic and a heart rate of $120 /$ min., so that they were not in fact harmed by our policy. (They both required only 2 units of blood acutely.)

To crossmatch two units of blood for each Caesarian section would increase the total crossmatches done in our blood bank by about 10 per cent (and about one-half of this workload would be on a stat basis in off hours). More units of blood would be tied up, the bank would have to order more units and the efficiency of blood utilization would decrease.

I feel that routine cross-matching is unwarranted when only 0.5 per cent of our sections need blood unexpectedly in the perioperative period. I cannot agree that the recommendation in the article represents the ideal form of blood management for Caesarian sections. In the absence of preoperative anaemia, bleeding or coagulopathy, a routine screen and hold is all that is necessary.

A.O. Davies, M.D., F.R.C.P.(C) North Bay, Ontario

\section{EPIDURal Morphine at Home}

\section{DEAR SIR,}

Epidural injection of morphine has recently been added to the armamentarium of the anaesthetist involved in the treatment of pain. This is proving to be a great boon post-operatively as well as in the care of terminal cancer.
I wish to submit a further advance using this modality - that is letting patients with terminal cancer go home with the epidural catheter securely taped in and covered. I permit the patient or his/her family to top-up the epidural morphine, provided by prescription.

So far I have two patients who have been happily at home with their families, not clouded by sedation, yet free of pain and able to move around freely, one for six weeks and the other for four weeks at this time.

The method is simple. An epidural catheter is installed at the level necessary for the pain in question. At the point of insertion, the skin is well sprayed with antibiotic and the whole is covered with "op-site" to seal air out and to hold the catheter in place. The distal end of the catheter is fixed over the shoulder of the patient and a micropore filter is fitted to the syringe fitting. I then inject morphine $5 \mathrm{mg}$ in $10 \mathrm{ml}$ of saline or water, without preservative. The patient is observed for approximately one hour and is then permitted to go home. They are given a prescription for an intravenous bag of distilled water or normal saline with morphine added to make $5 \mathrm{mg}$ in $10 \mathrm{ml}$. The patient, or his/her family, then withdraw $10 \mathrm{ml}$ of the solution and top-up the epidural when necessary. This usually amounts to every $10-12$ hours and thus the patient is kept free of pain.

I supervise these patients by frequent telephone calls, inspection in Emergency and replacement of the catheter when it is blocked or broken.

So far I find this a very satisfactory way of relieving pain for these terminally ill patients. J.M. Wishart, M.D., F.R.C.P.C.(C) Peterborough, Ontario. 